\title{
原子分辨显微分析技术研究进展
}

\author{
袁秉凯 ${ }^{1,2}$ 陈鹏程 ${ }^{1}$ 伅 君 ${ }^{1}$ 程志海 ${ }^{1}$ 装晓辉 ${ }^{1, *}$ 王 琛 ${ }^{1, *}$
}

('国家纳米科学中心, 北京 100190; 2 北京大学前沿交叉学科研究院, 北京 100871)

\begin{abstract}
摘要: 非接触原子力显微技术(NC-AFM)近年来发展迅速. NC-AFM 对单个分子的成像和谱学实现了原子分 辨和单个化学键分辨. NC-AFM 自身功能的拓展及其与不同探针技术的联用将为材料、物理、化学和生命科学 有关的研究提供崭新的思路. 本文首先介绍 NC-AFM 和 qPlus 传感器的基本原理, 然后讨论原子尺度的相互作 用力和短程力的精确测量, 总结近年来 NC-AFM 在原子尺度的化学结构成像、化学识别、电子结构性质分析以 及原子操纵技术中的研究进展, 并讨论了开尔文探针力显微技术(KPFM)在局域接触势差(LCPD)测量方面的 应用. 最后展望了NC-AFM面临的挑战和发展机遇.
\end{abstract}

关键词：扫描探针显微技术；非接触原子力显微技术； qPlus传感器；化学识别；原子操纵；电子结构; 开尔文探针力显微技术

中图分类号: 0647

\section{Research Progress in Atomic Resolution Microscopy}

\author{
YUAN Bing-Kai ${ }^{1,2} \quad$ CHEN Peng-Cheng ${ }^{1} \quad$ ZHANG Jun ${ }^{1} \quad$ CHENG Zhi-Hai ${ }^{1}$ \\ QIU Xiao-Hui ${ }^{1, *} \quad$ WANG Chen ${ }^{1, *}$
}

( ${ }^{1}$ National Center for Nanoscience and Technology, Beijing 100190, P. R. China; ${ }^{2}$ Academy for Advanced Interdisciplinary Studies, Peking University, Beijing 100871, P. R. China)

\begin{abstract}
Tremendous progress has been made in non-contact atomic force microscopy (NC-AFM) recently. The spatial resolution of NC-AFM imaging and spectroscopy of individual molecules on surfaces has reached true atomic resolution and bond differentiation level. Combination of NC-AFM with other scanning probe techniques can open a new way for materials, physics, chemistry, and biochemistry studies. In this review, we first introduce the basic principle of NC-AFM and qPlus sensor. The interaction force at atomic scale and precise measurement of short-range force are discussed. We summarize the recent advances in structural determination of organic molecules, chemical identification, electronic structure, and atomic manipulation at the atomic scale. In addition, we also discuss the application of Kelvin probe force microscopy (KPFM) in measurement of local contact potential difference (LCPD). Finally, perspectives and challenges in NC-AFM techniques are presented.
\end{abstract}

Key Words: Scanning probe microscopy; Non-contact atomic force microscopy; qPlus sensor; Chemical identification; Atomic manipulation; Electronic structure; Kelvin probe force microscopy

\footnotetext{
Received: January 14, 2013; Revised: April 17, 2013; Published on Web: April 19, 2013.

"Corresponding authors. WANG Chen, Email: wangch@nanoctr.cn; Tel: +86-10-82545609. QIU Xiao-Hui, Email: xhqiu@nanoctr.cn; Tel: +86-10-82545583.

The project was supported by the National Key Basic Research Program of China (2012CB933001) and National Natural Science Foundation of China (21173058).

国家重大科学研究计划(2012CB933001)和国家自然科学基金(21173058)资助

(C) Editorial office of Acta Physico-Chimica Sinica
} 


\section{1 引 言}

扫描探针显微技术(SPM) 是超高分辨显微技术 的重要分支, 它是 20 世纪 80 年代发展起来的一种 重要的表/界面探测技术. 1981 年 IBM (international business machines corporation)苏黎世实验室的科学 家 Binnig 等 ${ }^{1}$ 发明了扫描隧道显微镜(STM). 因其具 有极高的空间成像分辨率, 大量半导体或金属表面 的微观结构和物理化学性质得以在原子尺度上进 行研究. 利用 STM 的扫描隧道谱(STS)功能可以探 测分子的电子态、振动态以及自旋电子的相关信 息. 另外, STM 的原子/分子操纵功能可以构筑各种 功能化的纳米结构并可以原位进行其物理化学性 质的研究. 因而 STM 已成为表面科学研究中不可或 缺的技术手段. 但是 STM 以针尖与样品表面之间的 隧穿电流为探测信号, 需要样品导电, 因此不适于 绝缘材料表面的研究.

在早期的 STM 实验中, 研究者们发现针尖与样 品间距小至有隧穿电流产生时, 二者之间存在较强 的相互作用力. ${ }^{2,3}$ 1986年, Binnig、Quate 和 Gerber ${ }^{4}$ 以 针尖与样品之间的相互作用力作为探测样品表面 性质的信号, 发明了世界上第一台原子力显微镜 (AFM). 由于 AFM 探测的是针尖与表面之间的相互 作用力或者与力相关的物理量, 不需要样品导电, 因而可以用于研究更为广泛的材料体系. 目前, $\mathrm{AFM}$ 在物理、化学、材料、生物和工程等众多领域的 应用也越来越广泛深入.

AFM 有三种基本工作模式: 接触模式(contact mode)、振幅调制模式(amplitude-modulation mode)、 频率调制模式(frequency-modulation mode). ${ }^{5}$ 接触模 式 AFM, 又称静态 AFM, 以悬臂的偏转作为力的度 量, 在排斥力区域工作. 接触模式易于得到较高的 分辨率, 但针尖-样品之间作用力太大, 易损坏样 品. 振幅调制模式 $\mathrm{AFM},{ }^{6,7}$ 又称轻敲模式 AFM, 以振 幅或相位作为反馈信号对表面成像. 在轻敲模式 下, 悬臂受外力驱动在共振频率附近以恒定频率振 动, 振幅为一定值. 当针尖靠近样品时, 针尖 - 样品 之间作用力梯度发生变化, 引起悬臂共振频率的偏 移, 从而导致振幅和相位的变化, 通过反馈回路控 制振幅或者相位保持恒定, 可以获得样品的表面形 貌或者其他物理化学性质. 轻敲模式对样品的损坏 小, 适用于不同的材料, 是目前应用最广泛的 AFM 工作模式, 主要在大气和液相环境中使用. 由于在 轻敲模式下, 针尖-样品之间作用力引发的振幅和
相位的变化不是即时的, 其响应时间与品质因子成 正比. 而在超高真空环境下, 一般 AFM探针的品质 因子可大至 $10^{5}$, 轻敲模式 AFM 的信噪比得以提高, 但响应时间很长, 扫描速度很慢. 1991 年, Albrecht 等 ${ }^{8}$ 引入了频率调制模式. 频率调制模式 AFM, 又称 非接触原子力显微镜(NC-AFM), 测量针尖-样品之 间作用力梯度引起的悬臂共振频率的偏移 $(\Delta f)$. NC-AFM 是超高真空环境下 AFM 的主要工作模式. 随着技术的进步, NC-AFM不仅能够在非接触区域 工作, 还可以工作在排斥力区域.

超高真空环境下的 NC-AFM 在 AFM 各种工作 模式中具有最高的空间分辨率, 可以得到真正的原 子分辨图像. 近年来, 得益于 qPlus 传感器的引入, NC-AFM 的空间分辨取得了极大地提高, 能够得到 单个分子内的原子分辨甚至可以区分键级. ${ }^{910} \mathrm{NC}$ $\mathrm{AFM}$ 的力谱和三维力场成像功能可以在原子尺度 上实现针尖与表面特定位点之间的相互作用力和 能量的精确测量. . 另外, 能够在原子分辨成像的基 础上, 进行原子级的表面微区电特性的测量对于材 料性质、器件性能等至关重要. 近年来, NC-AFM 的 衍生技术一一开尔文探针力显微技术(KPFM)发展 迅速, 已经实现了原子尺度的表面电势甚至分子内 电荷分布的测量. ${ }^{12,13} \mathrm{NC}-\mathrm{AFM}$ 已经成为纳米科技领 域的重要研究工具..$^{14}$

本文首先介绍 NC-AFM 的基本原理; 然后讨论 原子尺度的针尖-表面之间的相互作用力以及频率 偏移与针尖-样品间作用力的关系; 在此基础上强调 小振幅 NC-AFM优势, 并由此介绍一种新型力传感 器— qPlus 传感器; 介绍短程力的精确测量和一种 表面三维化学力成像的方法; 最后介绍近年来超高 真空环境下的 NC-AFM 在原子尺度的化学结构成 像、化学识别、电子结构性质分析、原子操纵技术以 及高分辨 KPFM 中的若干应用, 并展望了这种技术 面临的挑战和发展机遇.

\section{2 非接触原子力显微镜的原理 \\ 2.1 基本工作原理}

AFM 的核心元件是测量针尖-样品间相互作 用力的传感器. 目前常见的力传感器是末端有探针 的微悬臂. 微悬臂的重要参数是弹性常数 $(k)$ 、自由 共振频率 $\left(f_{0}\right)$ 和品质因子 $(Q)$. 在频率调制模式下, 悬 臂受外力驱动以自由共振频率 $f_{0}$ 简谐振动, 振幅 $(A)$ 保持恒定. 当针尖逼近样品时, 针尖-表面之间的相 
互作用力梯度发生变化, 引起悬臂共振频率的偏 移. 测量共振频率偏移 $\Delta f$ 的方法主要有隧穿电流测 量、光束偏转技术、光纤干涉、压电和压阻等方式.

NC-AFM 的信号检测电路 ${ }^{15}$ (见图 1)主要由振幅 控制模块和频率测量模块两部分组成. 悬臂偏转信 号经带通滤波器滤去杂余频带信号, 分为三个支 路. 一路进入相位调节器. 相位调节器调节悬臂振 动激励信号与悬臂振动信号相位差 $\pi / 2$, 使激励信号 最小. 一路进入交流直流转换器. 交流直流转换器 将悬臂偏转振幅转化为直流信号, 并与振幅设定值 比较, 从二者的差值可以提取能量耗散信号. 交流 直流转换器和相位调整器共同构成振幅控制模块, 通过比例-积分-微分控制器(PID 控制器)控制振动 激励装置, 保持振幅恒定. 另一路进入基于锁相环 的频率调制解调器. 频率调制解调器测量频率偏移 信号, 并将其转化为电压信号. 在成像过程中, AFM 的扫描控制系统实时调控压电陶瓷扫描管, 从而调 整针尖与样品表面之间的距离以维持共振频率偏 移 $\Delta f$ 恒定, 记录压电陶瓷扫描管的运动轨迹即可得 到样品表面的形貌. 成像时也可关闭反馈, 保持压 电陶瓷扫描管与样品表面的距离不变, 记录频率偏 移信号, 得到样品表面与针尖之间作用力梯度的分 布.

\section{2 原子尺度的相互作用力分析原理}

真空环境下, AFM 针尖-样品之间的相互作用

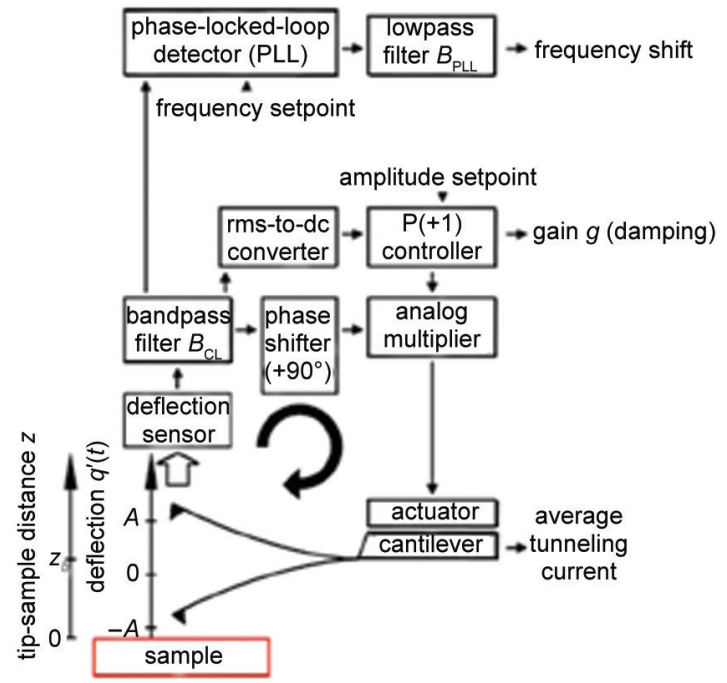

图 1 NC-AFM 的信号检测原理框图(恒定振幅控制和 频率偏移测量 $)^{15}$

Fig.1 Block diagram of the non-contact AFM feedback loop for constant amplitude control and frequency-shift measurement ${ }^{15}$
力包括吸引力和排斥力的贡献. 吸引力主要有范德 华力、静电力和化学吸引力, 排斥力主要是 Pauli 排 斥力. 对于磁性针尖或样品, 还有磁相互作用力的 存在. 一般而言, 原子尺度的范德华力是短程力, 但 是考虑到范德华力的加和性, 在靠近表面附近, 针 尖与表面之间的范德华力可视为长程力. 探针与样 品间的静电力是长程力 (可至 $100 \mathrm{~nm}$ ). 化学吸引力 和排斥力是短程力 ( $0.1 \mathrm{~nm}$ 量级). AFM 的成像分辨 率跟针尖的几何形状以及针尖-样品表面之间的相 互作用力密切相关. 在利用 AFM 获得原子分辨的图 像时, 一般认为成像的主要贡献是短程力, 而长程 力只是一个背景, 没有原子分辨的能力. 在半导体 表面, 原子分辨的获得是因为针尖与表面原子形成 局域的化学键. ${ }^{16}$ 在离子晶体表面, 不同电荷的原子 在表面周期排布产生随空间变化的静电势. 针尖与 针尖下方的离子之间的短程静电力作用, 是在离子 晶体表面得到原子分辨的主要原因. ${ }^{17}$ 分子的原子 分辨成像得益于针尖尖端原子与分子内的原子之 间的 Pauli 排斥作用..$^{18,19}$ 由于针尖和样品材料的功函 数不同, 针尖-表面之间会产生接触电势差, 为提高 微悬臂对短程力的灵敏度, 需要补偿接触电势差, 从而降低长程静电力的影响. 此外, 制备超尖针尖 可以减弱针尖-表面间的范德华力背景.

\section{3 原子尺度的相互作用力分析方法}

共振频率偏移 $\Delta f$ 是 NC-AFM 重要的可观测量, 反映的是悬臂振动周期内针尖-表面间相互作用力 $\left(F_{\mathrm{ts}}\right)$ 的权重平均值, $F_{\mathrm{ts}}$ 可看成是对悬臂简谐振动的 微扰. 微扰足够小时, $\Delta f$ 与 $F_{\mathrm{ts}}$ 有以下关系: $:^{20}$

$$
\Delta f=-\frac{f_{0}}{\pi k A_{0}} \int_{-1}^{1} F_{\mathrm{ts}}\left[z_{0}+A_{0}(1+u)\right]\left(\frac{-u}{\sqrt{1-u^{2}}}\right) \mathrm{d} u
$$

式中 $z_{0}$ 是振动周期内针尖 - 表面间的最小距离, $k$ 是 悬臂的弹性常数, $A_{0}$ 是悬臂的振幅. 由核函数(kernel function)

$$
K(u)=\left(\frac{-u}{\sqrt{1-u^{2}}}\right) \quad u \in\{-1,1\}
$$

可知, 悬臂在较大振幅下振动时, $\Delta f$ 主要由振动周 期内针尖与表面间距最近时 $(u=-1)$ 的作用力贡献; 在 $u=1$ 的发散点, 针尖-表面间作用力可以忽略不 计(图 2) ${ }^{20}$ 此时范德华力和长程静电力对 $\Delta f$ 有较大 贡献. 当悬臂振幅减小至与短程力衰减长度 (约为 $0.1 \mathrm{~nm}$ )可比时, $\Delta f$ 与针尖-表面间作用力的关系可 简化为: 
$\Delta f=k_{\mathrm{s} f} f_{0} / 2 k$

式中 $k_{\mathrm{ts}}$ 为针尖 - 表面弹性常数, 等于针尖 - 表面间相 互作用力梯度 $\left(-\mathrm{d} F_{\mathrm{ts}} / \mathrm{d} z\right)$. 相比长程力, 短程力梯度很 大, 对 $k_{\mathrm{ts}}$ 贡献也大. 所以悬臂以小振幅振动时对短 程力尤为敏感, 而长程力对 $\Delta f$ 贡献变小.

硅材料悬臂的弹性常数一般为几个 $\mathrm{N} \cdot \mathrm{m}^{-1}$ 到几 十 $\mathrm{N} \cdot \mathrm{m}^{-1}$, 振幅为几纳米至几十纳米. 如以 $0.1 \mathrm{~nm}$ 量 级振幅振动, 当针尖与表面吸引力大过悬臂恢复力 时, 针尖易发生“突跳” (jump to contact)现象. ${ }^{21}$ 悬臂 要以 $0.1 \mathrm{~nm}$ 量级振幅稳定振动, 弹性常数需要大至 几百 $\mathrm{N} \cdot \mathrm{m}^{-1}$. 因悬臂以小振幅振动可以部分消除长 程力对成像的影响, 研究者们发展了多种亚纳米振 幅的 NC-AFM技术, 主要是基于高弹性常数的石英 音叉和长度伸缩模石英谐振器 (quartz LER) ) $^{22,23}$, 其中 前者主要是 qPlus 传感器 ${ }^{24}$ 和双石英音叉传感器. ${ }^{25}$ LER 和石英音叉均由两个耦合的具有相同共振频 率并以反平行方式振动的电机械振子组成. ${ }^{26}$ LER 以振动时的压电信号测量频率偏移 $\Delta f$, 属于自检测 力传感器. 弹性常数 $k$ 为几万 $\mathrm{N} \cdot \mathrm{m}^{-1}$, 共振频率为 $0.5 \mathrm{MHz}$ 到几个 MHz. 2008年, Hasegawa 等 ${ }^{23}$ 利用基

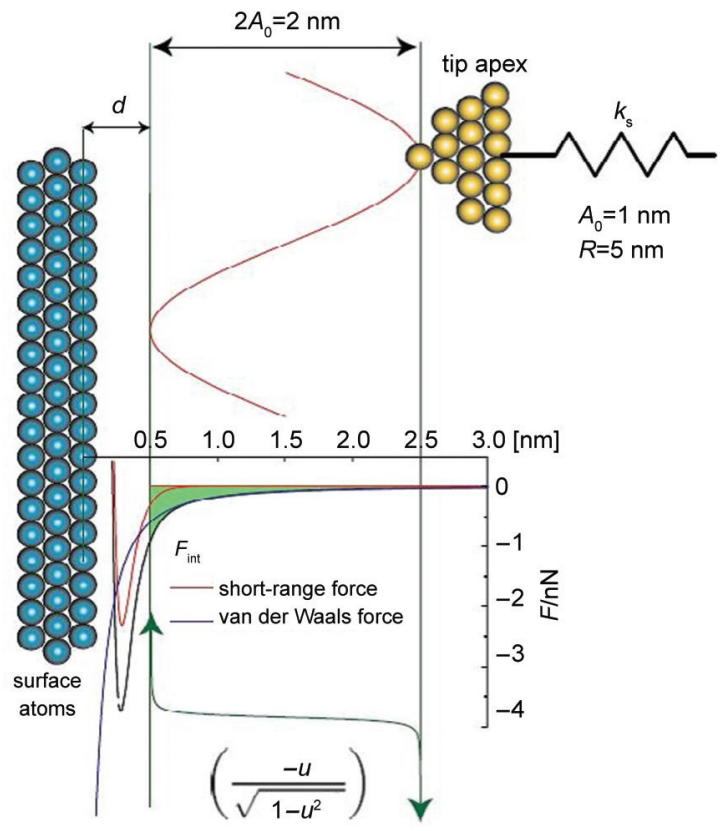

图 2 探针尖端与表面的总相互作用力以及 核函数 $K(u)$ 的形状 ${ }^{20}$

Fig.2 Total interaction force between tip apex and surface and the behavior of the kernel function $K(u)^{20}$

$$
K(u)=\left(\frac{-u}{\sqrt{1-u^{2}}}\right) \quad u \in\{-1,1\}
$$

$k_{\mathrm{s}}$ : spring constant of the cantilever; $A_{0}$ : the amplitude of the cantilever; $R$ : the nominal radius of the tip apex; $d$ : the closest tip-surface distance; $F_{\text {in: }}$ the interaction force
于 LER 的 AFM 以几十 $\mathrm{pm}$ 的振幅得到了 $\mathrm{Si}(111)-(7 \times$ 7)表面的原子分辨. qPlus 传感器由雷根斯特堡大学 Giessibl教授发明, 近年来得到了广泛的应用并取得 了令人瞩目的成果. qPlus 传感器以石英音叉为力传 感器并利用石英的压电效应测量频率偏移 $\Delta f$, 属于 自检测力传感器. 根据所选用的石英音叉, 弹性常 数 $k$ 约为几千 $\mathrm{N} \cdot \mathrm{m}^{-1}$, 共振频率为 $32-200 \mathrm{kHz}$. 图 3 是 qPlus 传感器的光学显微镜照片. 其中石英音叉 的一支悬臂固定于底座, 探针粘在另一支悬臂上. 而 LER 中针尖沿长轴方向粘在石英条的一边, 形成 针形传感器. 与激光检测的 $\mathrm{Si}$ 悬臂相比, qPlus 传感 器和 LER 具有以下优势: $:^{15,27}$ 悬臂的弹性常数一般可 达几千 $\mathrm{N} \cdot \mathrm{m}^{-1}$ 甚至几万 $\mathrm{N} \cdot \mathrm{m}^{-1}$, 悬臂可以亚埃振幅 稳定振动, 对短程化学力和 Pauli 排斥作用更加敏 感, 分辨率更高; 无激光检测, 因而低温实验时没有 激光产生的热噪声; 沿特定方向切割的石英晶体其 共振频率的温度漂移小于 $\mathrm{Si}$ 悬臂; 石英音叉较大, 仅用镊子和光学显微镜就可在音叉上安装探针, 故 探针制备简单, 价格低廉, 可选材料广泛. 此外, 基 于 qPlus 传感器和 LER 的 AFM 易与 STM 功能结合. 这种双模式的 STM/AFM 可以同时获取针尖-表面 相互作用力和隧穿电流的信息. 比较两种技术采集 的数据特征和差异, 可以得到丰富的表面结构和样 品电子态信息.

\section{4 原子尺度的相互作用力定量测量}

NC-AFM 的谱学功能可以研究材料的不同性 质. 力谱可以定量测量针尖-表面之间的相互作用

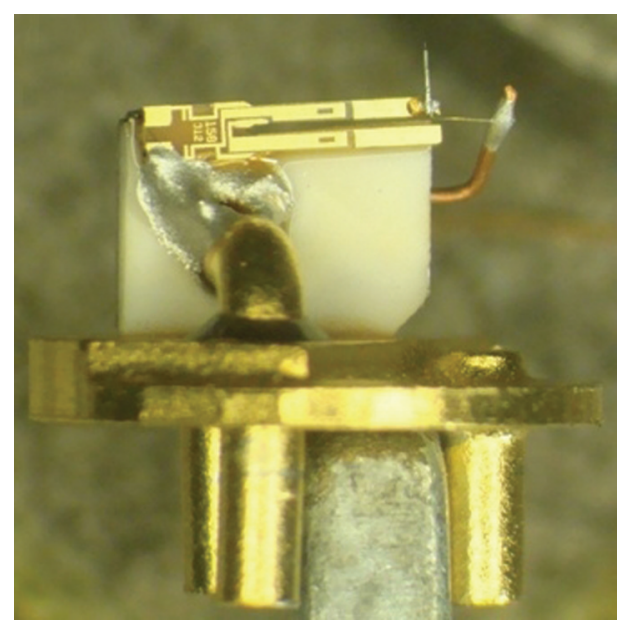

图 3 qPlus 传感器显微照片

Fig.3 Micrograph of a qPlus sensor

The qPlus sensor consists of three parts: a tuning fork, a ceramic substrate, and a tip. The tip is connected with a squeezed gold wire (diameter $12.7 \mu \mathrm{m}$ ) in order to measure tunneling current. 
力和能量, 是研究高分辨成像和原子/分子操纵机理 的关键. 力谱是在特定的位置上记录针尖-表面相 互作用力梯度 (即频率偏移量 $\Delta f$ )与针尖-表面间距 (z) 的关系, 即 $\Delta f(z)$ 曲线. 记录 $\Delta f(z)$ 曲线时, 针尖至少 从悬臂自由振动位置逐渐逼近表面到短程力产生 或者继续逼近至排斥力区域. 记录 $\Delta f(z)$ 曲线时, 探 针需在较长时间内以原子精度定位于所测原子或 分子上, 但热漂移会使针尖在 $X 、 Y$ 方向偏离所测原 子, 降低谱学测量的空间分辨率. 实验中可以采用 低温实验或者利用控制软件进行漂移补偿的方法 来降低热漂移对力谱测量的影响.

力谱是基于公式(1)实现的. 反演公式(1)可以得 到针尖-表面之间的相互作用力以及势能与针尖表面间距的关系, 即 $F_{\mathrm{ts}}(z)$ 和 $E_{\mathrm{ts}}(z)$ 曲线. 目前 Sader 和 $\mathrm{Jarvis}^{28}$ 提出的方法准确而简单易行, 使用较为广泛. 计算得到的针尖-表面间的相互作用力包含长程力 和短程力. 由于高分辨成像、表面原子/分子操纵、化 学元素识别等利用的主要是短程力, 所以短程力的 精确测量非常重要. 从计算得到的总相互作用力中 扣除长程力, 可以得到短程力, 而长程力主要有静 电力和范德华力. 采集力谱时, 静电力可通过偏压 补偿, 所以如何有效扣除范德华力是关键. 在表面 特殊位点(如空位)记录 $\Delta f(z)$ 曲线至短程力出现, 无 空位等特殊位点的表面上测得的力谱共同部分即 可认为是范德华力. 根据 Hamaker 方法 ${ }^{29}$ 假设的范 德华力的可加和性, 靠近表面、半径为 $R$ 的球形针 尖与表面之间的范德华力正比于 $1 / z^{2},{ }^{30,31}$ 拟合后可 以得到范德华力 $F_{\mathrm{vdw}}(z)$ 曲线. 从总相互作用力中扣 除范德华力可得到短程力与针尖-表面间距的关系 $F_{\text {short-range }}(z)$. 利用这种方法不仅可以精确测定针尖-表 面之间的相互作用力, 而且可以得到不同类型相互 作用力的贡献, 从而为理解成键、表面化学反应、表 面催化、表面吸附和扩散等过程奠定了基础.

在三维空间记录密集高质量的 $\Delta f(x, y, z)$ 可以实 现表面三维化学力成像. ${ }^{1}$ 表面三维化学力成像对 诸多领域, 如催化、薄膜生长、摩擦力等研究有重要 意义. 这种测量可以确定吸附原子在表面的扩散势 垒, 包括缺陷对扩散的影响. 表面三维力场测量与 理论模拟的比较能从定性和定量的角度研究针尖 尖端靠近表面, 尤其是进入排斥力区域时发生的原 子结构弛豫对相互作用力和势能的影响. ${ }^{32-36}$ Albers 等 ${ }^{37,38}$ 展示了一种测量表面三维化学力成像的方法. 他们缓慢改变 $\Delta f$ 设定值, 至针尖振动不受表面影
响, 在不同 $\Delta f$ 下逐帧(image-by-image)扫描得到形貌 像并采集数据, 并在数据处理时调整图像间相对位 置, 补偿针尖-样品间的漂移, 截取所有图像均重合 的部分, 获得 $\Delta f(z)$ 谱线的分布图. 所记录的 $\Delta f(z)$ 谱线 可以转化为 $F_{\mathrm{ts}}(z)$ 或者 $E_{\mathrm{ts}}(z)$. 所有谱线集合在一起就 成为 $F_{\mathrm{ts}}(x, y, z)$ 或者 $E_{\mathrm{ts}}(x, y, z)$ 阵列. 沿不同方向在三 维空间切一平面, 可以得到表面形貌、力场空间分 布等信息. 这种方法可以在数据处理时校正漂移的 影响, 而且容易察觉数据采集过程中针尖状态的变 化.

\section{3 原子尺度的原子力显微方法应用 \\ 3.1 原子尺度的化学结构成像}

在实空间 “目睹”分子中的原子和化学键一直 以来都是化学家的梦想. STM 能够获得各类晶体材 料表面的原子分辨图像. STM 检测的隧道电流对费 米面附近的电子态密度敏感, 因此 STM 能够对分 子轨道成像, 却很难对分子内的原子结构成像. 最 近由 Temirov 与其合作者 ${ }^{39-42}$ 发展的扫描隧道氢显 微技术(scanning tunneling hydrogen microscopy)实 现了分子内的原子分辨. 1995 年, Giessibl ${ }^{43}$ 首次利 用 NC-AFM 在 $\mathrm{Si}$ 表面获得原子分辨图像. 此后, 多 种半导体表面( $\mathrm{Si}(100)-(2 \times 1) 、 \mathrm{GaAs}(110) 、 \operatorname{InP}(110)$ 、 $\operatorname{InAs}(110)$ 等)和高定向热解石墨(HOPG)的原子分辨 成像均有报道; 在绝缘材料 $(\mathrm{NaCl} 、 \mathrm{MgO}$ 等)和金属 单晶 $(\mathrm{Cu}(100) 、 \mathrm{Ag}(100)$ 等)表面也都获得了原子分辨 图像; $\mathrm{C}_{60}$ 、硫醇等分子分辨成像的研究也有很多报 道. ${ }^{44,45}$

最近, 得益于 qPlus 传感器应用, NC-AFM 得到 了较STM更高的空间分辨率, 实现了有机分子内的 原子和化学键成像, 甚至区分化学键的键级. Giessibl 与其合作者 ${ }^{46,47}$ 得到了 $\mathrm{Si}(111)$ 表面原子的亚 原子分辨图像和 $\mathrm{W}$ 探针尖端的电荷分布. 2009 年, IBM 苏黎世实验室的 Gross 等 ${ }^{48}$ 在液氦温度、横高模 式下扫描得到了并五苯分子的原子分辨像, 这是第 一次利用 AFM 获得的分子的化学结构成像. 图 4(a) 是吸附在 $\mathrm{Cu}(111)$ 表面上的并五苯分子的 STM 图 像, 图 4(b-f) 是并五苯分子的 AFM 频率偏移图像. 由于分子中共轭电子的离域性, STM 图像中观察不 到分子的精细结构. 而在 AFM图像中并五苯分子的 五个六边形碳环结构都能清楚分辨, 甚至能够对 $\mathrm{C}-\mathrm{H}$ 键成像. 作者进一步研究了 $\mathrm{CO}$ 修饰的针尖与 并五苯分子的相互作用力, 实验和理论计算结果表 
明, ${ }^{18}$ 针尖与分子间的 Pauli 排斥力是得到分子化学 结构成像的主要原因, 范德华力及静电相互作用力 表现为在整个分子上均匀分布的吸引力背底. 针尖 与分子间 Pauli 排斥力反映了总电子态密度, 因而能 对分子的骨架成像. 因此实现分子的原子分辨, 需 要在排斥力范围对分子成像, 为了提高对短程力 (Pauli 排斥力) 的灵敏度, 图像是悬臂以 $100 \mathrm{pm}$ 左右 振幅振动时获得的. 目前, 获得分子内原子分辨的 qPlus 传感器所用振幅在 30-100 pm 之间. 另外, 针 尖的原子级功能化修饰也是分子内的原子分辨成 像的关键, 这是通过可控的垂直原子/分子操纵实现 的. 目前主要是 $\mathrm{CO}$ 分子修饰的针尖. $\mathrm{CO}$ 分子的化 学惰性使得针尖可以稳定地进入排斥力区域而不 与待研究分子成键. 金属针尖通常很难在排斥力区 域对分子进行稳定的成像, 但是 Pawlak 等 ${ }^{49,50}$ 利用 金属针尖在排斥力区域得到了 $\mathrm{C}_{60}$ 分子的原子分辨 图像, 并据此确定了 $\mathrm{C}_{60}$ 分子在表面的吸附取向. Boneschanscher 等 ${ }^{51}$ 研究了金属和 $\mathrm{CO}$ 针尖对石墨烯 成像的机理, 并成功分辨石墨烯的边界构型, 为进 一步研究石墨烯的缺陷、边界等结构对其性质的影 响打下基础.

利用 NC-AFM 对分子进行化学结构成像, 进一 步提高分辨率并挑战分辨率的极限是科学家追求 的一个目标, 另一个追求的方向是高分辨成像在结 构解析方面的应用, 特别是难于进行结晶结构解析
的分子. 在进一步提高分辨率方面, 最近 Gross 等 ${ }^{10}$ 利用 $\mathrm{CO}$ 针尖测量了多环芳烃和富勒烯分子中不同 $\mathrm{C}-\mathrm{C}$ 键电荷分布和键长的细微差别, 区分了不同 $\mathrm{C}-\mathrm{C}$ 键的键级(图 4(i,j)). 不同 $\mathrm{C}-\mathrm{C}$ 键反差产生主 要有两个来源: (1) 键级高的 $\mathrm{C}-\mathrm{C}$ 键电子态密度 高, 与针尖的排斥力大; (2) 针尖上吸附的 $\mathrm{CO}$ 分子 的倾斜 ${ }^{35}$ 放大了不同 $\mathrm{C}-\mathrm{C}$ 键键长的差别. 键级的区 分使得缺陷结构以及缺陷引发的细微电荷分布变 化的研究成为可能. . $^{52}$ 在结构解析方面, Gross 等 ${ }^{33}$ 利 用 qPlus 技术对有机分子的化学结构成像, 与核磁 共振实验和理论模拟相结合, 实现了天然产物头孢 菌素类化合物 (cephalandole A) 结构的测定 (图 4(g, h)), 这对于新药的开发和笁选意义重大.

原子之间形成的共价键具有很强的方向性, 过 渡金属与配体成键的方向性尤其明显. 最近, Welker 和 Giessib ${ }^{54}$ 利用 qPlus 技术的三维力谱功能研究了 针尖与 $\mathrm{CO}$ 分子间相互作用随二者距离和角度的变 化关系, 揭示了金属针尖与吸附在 $\mathrm{Cu}(111)$ 表面的 $\mathrm{CO}$ 分子间化学键力的角度依赖性. 结合理论模拟, 他们提出了一个键能的角度依赖模型, 并指明针尖 与分子间角度依赖的相互作用势的快速衰减是成 像反差的来源.

STM 和 NC-AFM 测量不同的性质, 基于 qPlus 传感器的 SPM 系统可以方便地实现二者的结合, 从 而提供结构和电子态的互补信息. Mohn 等 ${ }^{55}$ 利用

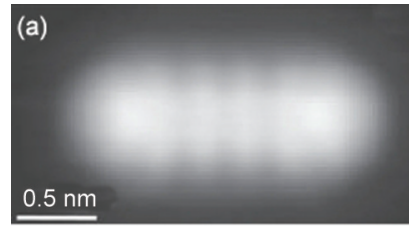

(c)

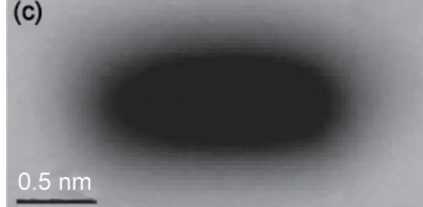

(e) (b)

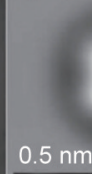

(d) $0.5 \mathrm{~nm}$
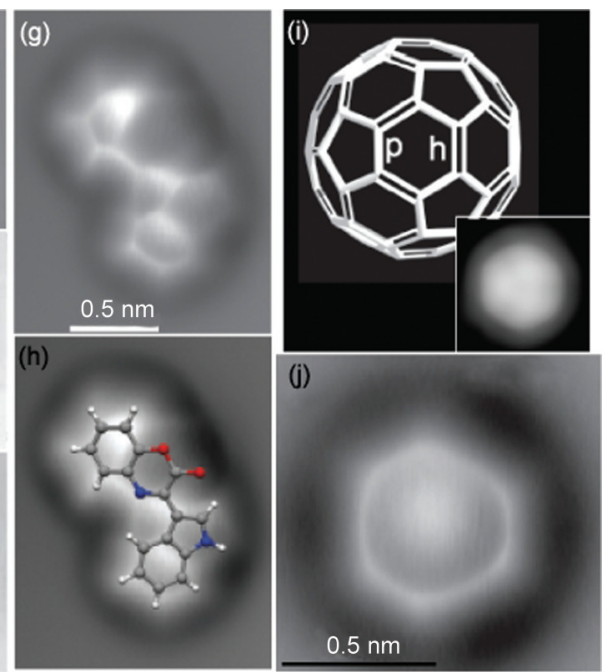

图 4 单分子内的 NC-AFM 原子分辨成像 ${ }^{10,48,53}$

Fig.4 NC-AFM images of molecules with atomic resolution ${ }^{10,48,53}$

(a) STM image of pentacene on $\mathrm{Cu}(111)$. (b) constant-height AFM image of pentacene on $\mathrm{Cu}(111)$ with $\mathrm{CO}$ tip. (c-f) constant-height AFM images of pentacene on $\mathrm{NaCl}(2 \mathrm{ML}) / \mathrm{Cu}(111)$ with different tip terminations, (c) Ag tip, (d) $\mathrm{CO}$ tip, (e) Cl tip, (f) pentacene tip. (g, h) constant-height AFM image of cephalandole A and the same image with superimposed molecular model, respectively. (i, j) molecular model and the constant-height AFM image of $\mathrm{C}_{60}$, the inset in (i) is the STM image of $\mathrm{C}_{60}$. 
STM 和 NC-AFM 在 $\mathrm{NaCl}(2 \mathrm{ML}) / \mathrm{Cu}(111)$ 表面研究了 菲四甲酸二䣶(PTCDA)分子与单个 $\mathrm{Au}$ 原子可逆成 键形成的分子开关. 他们利用 STM 操纵这种分子开 关并表征其电子态性质, NC-AFM成像则确定了 $\mathrm{Au}$ 原子/分子配合物开关状态的化学结构. Pavliček 等 ${ }^{56}$ 利用 STM 实现了立体分子噻蒽衍生物(dibenzo[1,8] thianthrene, DBTH) 在 $\mathrm{NaCl}(2 \mathrm{ML}) / \mathrm{Cu}(111)$ 表面的两 种吸附构型间的可逆转变. NC-AFM 成像得到了 DBTH 分子两种吸附构型的空间结构, 由此可以推 断出构型转变的途径. 另外, STM 和 NC-AFM 的成 像结果的比较也加深了对 STM 成像机制的理解.

综上所述, 利用 NC-AFM 对分子内原子和化学 键的高分辨成像可以研究分子在表面的几何构型 和原子间成键特性, 如键级、键长等. 结合谱学功 能, 探测分子不同位点的物理和化学特性成为可 能. 目前, NC-AFM 多用于平面分子的原子分辨成 像, 探针修饰主要依靠 CO 分子, 这局限了 NC-AFM 的应用范围. 随着探针修饰方法的发展, 如各种有 机配体或者酸碱基团修饰的探针的出现, NC-AFM 的研究体系和领域将得到极大的拓宽, 局域化学环 境下表面活性位点或者缺陷的化学反应活性可以 得到更加深入和细致的研究, 从而使人们能够在原 子水平上理解化学反应与催化的机理. 由于表面吸 附的稳定作用, 一些亚稳态的化学反应过渡态的实 空间观测也将使得对化学反应、催化机理及其动力 学过程的理解达到更深的层次.

\section{2 原子尺度的化学识别}

表面原子/分子的化学识别一直是扫描探针显 微技术的目标. STM 的谱学功能被广泛用于这方面 的研究. Ho 与其合作者 ${ }^{57}$ 利用亚分子分辨的非弹性 电子隧道谱区分了吸附在 $\mathrm{Cu}(100)$ 表面上的单个乙 炔和氛代乙炔分子的 $\mathrm{C}-\mathrm{H}$ 和 $\mathrm{C}-\mathrm{D}$ 伸缩振动. 近 年来 NC-AFM 在表面化学识别方面取得了突破性 的进展. NC-AFM 已经可以分辨半导体 ${ }^{58,59}$ 和绝缘 体 ${ }^{60-63}$ 表面以及有机分子内 ${ }^{10,48,53,55}$ 的化学组分. 值得 注意的是, 化学元素识别可以通过测量特定位点针 尖-表面原子间相互作用力实现.

离子晶体表面不同位点的力谱与理论模拟的 比较可以确定表面不同离子的亚晶格和针尖的化 学成分. ${ }^{60,61,63}$ 半导体表面的元素化学识别可以通过 测量针尖与表面原子形成的局域化学键实现. 2001 年 Lantz 等 ${ }^{64}$ 首次在低温下利用力谱测量了针尖与 $\mathrm{Si}(111)-(7 \times 7)$ 表面 $\mathrm{Si}$ 增原子间形成的共价键力的大
小. 2007 年, Sugimoto 等 ${ }^{59}$ 在室温下利用力谱测量针 尖-表面原子间的化学键力, 实现了 $\mathrm{Si} 、 \mathrm{Sn}$ 和 $\mathrm{Pb}$ 合 金表面组分的化学识别(图 5). 几种原子的“化学身 份”无法从形貌和高度图中区分, 但是通过测量单 个原子与针尖的化学键力大小, 他们发现不同种类 的原子一针尖之间的相互作用力有显著差异. 同种 类原子与不同状态针尖间相互作用力差别很大(如 图 5(a,c)所示), 但是对于相同状态的针尖, 不同种 类表面原子与针尖的最大吸引力之间的比值为定 值(如图 5(b,d) 所示), $\mathrm{Pb}$ 和 $\mathrm{Si}$ 原子与针尖最大吸引 力的比值为 0.59 , 而 $\mathrm{Sn}$ 和 $\mathrm{Si}$ 原子与针尖最大吸引力 的比值为 0.77 . 根据针尖与不同原子间最大吸引力 的比值, 可以实现表面 $\mathrm{Si}, \mathrm{Pb}$ 和 $\mathrm{Sn}$ 原子的化学识别, 从而测量局域环境下不同原子的分布(图 5(e, f)). Setvín等 ${ }^{58}$ 将表面元素的化学识别推广至 III-IV 族
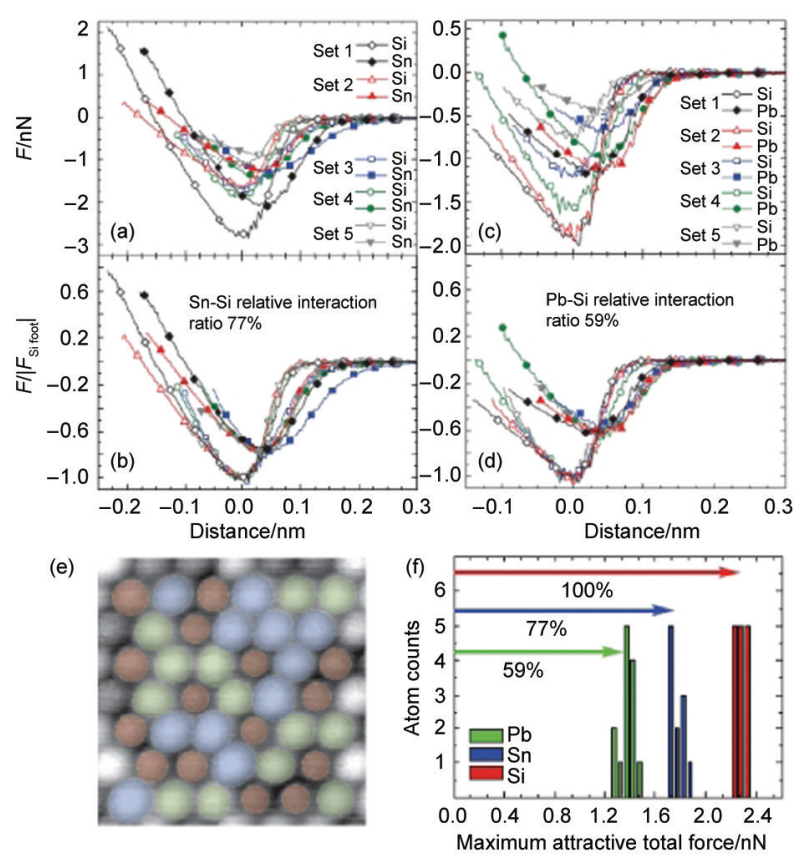

图 5 通过探测短程化学相互作用力化学识别 表面单个原子 ${ }^{59}$

Fig.5 Chemical identification of individual surface atoms by probing short-range chemical interaction forces ${ }^{59}$

(a) sets of short range force curves obtained over structurally equivalent $\mathrm{Sn}$ and $\mathrm{Si}$ atoms. The tips differ from set to set. (b) the same force curves as in (a) with the curves in each set normalized to the absolute value of the minimum short range force of the Si curve. (c, d) sets of short-range force curves for $\mathrm{Pb}$ and $\mathrm{Si}$, before (c) and after (d) normalization. (e) NC-AFM image showing the local chemical composition of a surface alloy composed of $\mathrm{Si}, \mathrm{Sn}$ and $\mathrm{Pb}$ atoms blended in equal proportions on a $\mathrm{Si}(111)$ substrate. Green, blue, and red atoms correspond to $\mathrm{Pb}, \mathrm{Sn}$ and $\mathrm{Si}$, respectively. (f) atom counts as a function of the maximum attractive total force measured over the $\mathrm{Pb}, \mathrm{Sn}$ and $\mathrm{Si}$ atoms. 
元素, 在 $\operatorname{Si}(100)-(2 \times 1)$ 表面实现了 $\mathrm{In}$ 和 $\mathrm{Sn}$ 元素的识 别, 并指出同种元素的不同价态也可以区分.

目前, 有机分子内的化学识别仍面临几个挑战: 有机分子 $\mathrm{C}-\mathrm{C}$ 键长远短于硅原子最近邻间距; 分 子的立体构型使得数据分析和解释变得更加复杂; 杂化分子轨道与针尖没有悬挂键作用; 与晶格原子 相比, 有机分子在表面吸附不稳定, 成像时易被操 纵, 尤其在绝缘材料表面上. 但是, 半导体表面元素 化学识别和区分 $\mathrm{C}-\mathrm{C}$ 键键级的工作为有机分子内 的化学识别带来了希望. 可能的思路是通过针尖功 能化来提高化学识别能力, 不同功能化的针尖与特 定元素或者官能团之间的相互作用可能产生化学 反差, ${ }^{65}$ 从而实现有机分子内的化学识别. STM 和 NC-AFM 的联用也将有助于这一问题的研究.

\section{3 原子尺度操纵技术}

扫描探针显微技术除了可以对表面进行成像和 性质测量, 还可以进行表面原子/分子的操纵. 原子/ 分子操纵技术于 1990 年由 IBM 公司的 Eigler 等 ${ }^{66}$ 首 次发明. 他们利用 STM 在 Ni(110)表面对单个Xe原 子进行操纵. 探针可以推、拉或者滑动(push, pull or slide)表面上的原子和分子, ${ }^{67}$ 这是横向操纵技术; 探 针还可以捡起和放下原子和分子, ${ }^{68,69}$ 这是纵向操纵 技术. 横向操纵技术可以将不同原子和分子操纵至 特定位点, 构筑有特定功能的纳米结构, 如量子围 栏 ${ }^{70}$ 和人造分子 ${ }^{71}$ 等; 纵向操纵技术可以用于原子尺 度的接触研究, 还可以用于表面原子和分子对针尖 的修饰, 这对于超高分辨成像至关重要. 近几年来, 基于 $\mathrm{AFM}$ 的原子操纵技术取得了长足的进步, 已被 用于半导体表面的原子图案设计、绝缘材料表面的
原子操纵等. ${ }^{72,73}$ 与 STM 操纵技术相比, AFM 操纵技 术尚处于初期阶段, 但已初步展现了研究纳米尺度 基本问题的强大能力和重要性.

半导体表面的操纵主要是由日本大阪大学的 Morita 研究组实现的. 由于原子/分子与半导体表面 原子的成键作用, STM 在半导体表面的操纵实验一 般是利用电子或者非弹性共振隧穿来实现的, 而半 导体表面的 AFM 操纵一般是纯机械操纵. 2003 年, Morita 研究组 ${ }^{74}$ 利用 NC-AFM 首次实现了 Si(111)$(7 \times 7)$ 表面增原子的机械垂直可逆操纵. Morita研究 组 ${ }^{75}$ 发现室温下 $\operatorname{Si}(111)-(7 \times 7)$ 表面增原子的横向操 纵在较小的短程力区域即可实现. 他们 ${ }^{76,77}$ 还利用 NC-AFM 在 Ge(111) $-c(2 \times 8)$ 表面实现了单个原子的 纵向和横向操纵, 并在室温下通过 AFM 针尖引发 $\mathrm{Ge}(111)-c(2 \times 8)$ 表面置换的 $\mathrm{Sn}$ 原子与邻近 $\mathrm{Ge}$ 原子 的交换实现了原子图案的构造(图 6(d)). 这种横向操 纵是通过针尖-表面之间吸引力和针尖扫描方向的 精确控制来实现的. 图6(a)是表面原子横向交换操 纵过程示意图. 首先得到表面的原子分辨图像(图 6 (b)), 其中 $\mathrm{Sn}$ 原子较 $\mathrm{Ge}$ 原子祄度高. 选择如图 6(b) 中蓝色方框所示的小区域进行扫描, 扫描角度与待 操纵的 $\mathrm{Sn}$ 原子和 $\mathrm{Ge}$ 原子向量方向一致. 针尖扫描 至连接两原子中间的线时(图 6(b)蓝色箭头处), 停止 慢扫描方向的扫描. 然后固定扫描同一条线, 逐渐 降低针尖-表面间距, 增加针尖尖端原子与表面的吸 引力. 线扫描过程中, 吸引力超过 $\mathrm{Sn}$ 原子和 $\mathrm{Ge}$ 原子 交换所需作用力的阈值时, 两原子发生交换. 操纵 时的针尖-表面间的相互作用力与针尖尖端原子和 表面吸附原子间的共价键有关. 按照径向原子交换
(1)

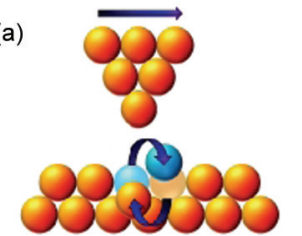

(e)

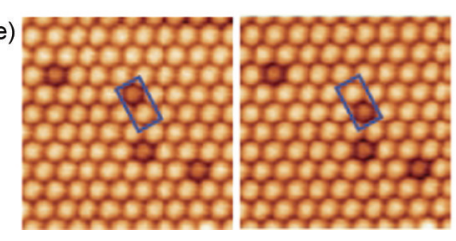

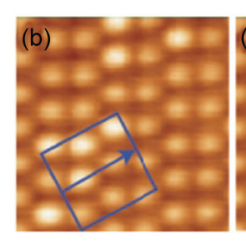
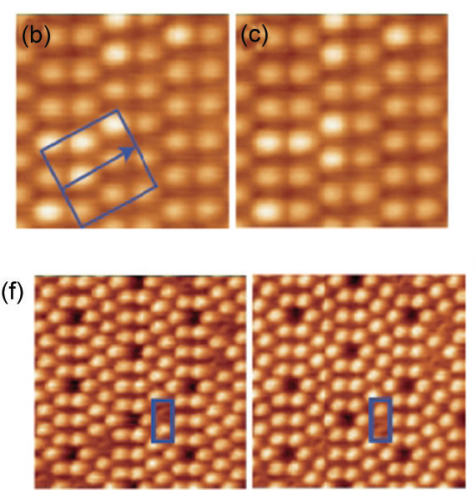

图 6 半导体表面的横向交换操纵 ${ }^{72}$
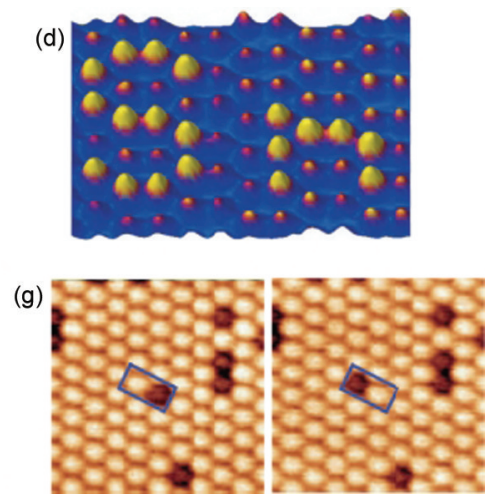

Fig.6 Lateral interchange manipulation on semiconductor surfaces ${ }^{72}$

(a) schematic illustration of lateral interchange manipulation. (b, c) AFM images before and after the lateral manipulation of Sn atoms (higher protrusions) on the $\mathrm{Ge}(111)-c(2 \times 8)$ surface, and the scan direction is highlighted in (b) by the arrow in the blue square. (d) final atomic design after lateral interchange manipulation. Identical method has been successfully applied in other surfaces of different structure and composition.

(e) $\mathrm{Sn} / \mathrm{Si}(111)-(\sqrt{3} \times \sqrt{3}) R 30^{\circ}$, (f) $\mathrm{In} / \mathrm{Si}(111)-(\sqrt{3} \times \sqrt{3}) R 30^{\circ}$, (g) $\mathrm{Sb} / \mathrm{Si}(111)-(7 \times 7)$ 
操纵方法, 可得到如图 6(d) 所示的原子设计图案. 这 种横向交换操纵的方法具有普遍性, 可以在不同结 构和组成的表面实现, ${ }^{72,78}$ 如 $\mathrm{Sn} / \mathrm{Si}(111)-(\sqrt{3} \times \sqrt{3})$ $R 30^{\circ}, \mathrm{In} / \mathrm{Si}(111)-(\sqrt{3} \times \sqrt{3}) R 30^{\circ}$ 和 $\mathrm{Sb} / \mathrm{Si}(111)-(7 \times 7)$ (图 $6(\mathrm{e}-\mathrm{g}))$. 另外, Morita 研究组 ${ }^{79}$ 还在 $\mathrm{Sn} / \mathrm{Si}(111)-(\sqrt{3} \times$ $\sqrt{3}) R 30^{\circ}$ 表面利用探针尖端 $\mathrm{Si}$ 原子与表面 $\mathrm{Sn}$ 原子之 间的可逆垂直交换, 通过依次沉积 $\mathrm{Si}$ 原子至表面的 方法创建出原子级的图案结构, 实现了“原子写字” 的功能.

与在金属和半导体表面的吸附不同, 原子/分子 与绝缘体表面的相互作用小, 易于在表面扩散, 操 纵时也容易吸附到针尖上. ${ }^{80}$ 一般绝缘材料表面的 操纵较半导体表面的操纵困难, 主要是在低温下实 现的, 研究较少. 但已有的研究表明了 NC-AFM 具 有绝缘材料表面操纵原子/分子的能力. 2006 年, Hirth 等 ${ }^{81}$ 在室温下于 $\mathrm{CaF}_{2}$ (111) 表面成功实现了原 子尺度缺陷的横向操纵. 几乎同时, Morita 研究组 ${ }^{82}$ 在 $\mathrm{KCl}(100)$ 表面得到了相似的空位缺陷横向操纵 的结果. 最近他们 ${ }^{83}$ 报道了离子晶体表面单原子缺 陷随针尖连续移动过程中的随机跳跃现象. 因为频 率偏移的设定值与针尖/缺陷间较弱的相互作用引 起的频率偏移差异较大, 他们将这一现象归处于频 率偏移差值引起的过反馈. 绝缘材料表面的操纵需 要更加精细地控制针尖、表面与待操纵的原子/分子 之间相互作用力的平衡, qPlus 传感器的亚埃振幅将 有助于绝缘材料表面原子/分子操纵的精确控制.

利用针尖 - 样品间相互作用力的精确测量, NC-AFM 还可以研究原子/分子操纵的机理, 分析并 拟合分子操纵过程的力谱, 可以定量得到分子在样 品表面的总吸附能, 并确定不同的分子-表面间的 相互作用对吸附能的贡献. 在 STM 横向操纵实验 中, 表面吸附的原子或分子与探针针尖形成化学 键, 随针尖从表面上的一个吸附位跃至另一个吸附 位, 但是操纵过程中探针与吸附原子或分子间的相 互作用力却难以定量测量. 2008 年, Ternes 等 ${ }^{84}$ 首次 定量测量了沿表面拉动单个原子或分子所需力的 大小. 实验发现在 $\mathrm{Pt}(111)$ 表面拉动 Co 原子需横向 力 $210 \mathrm{pN}$, 在 $\mathrm{Cu}(111)$ 表面拉动 $\mathrm{Co}$ 原子的横向力仅 为 $17 \mathrm{pN}$, 横向力的大小与施加在 $\mathrm{Co}$ 原子上的垂直 方向的力无关. 在 $\mathrm{Cu}(111)$ 表面操纵 $\mathrm{Co}$ 原子和 $\mathrm{CO}$ 分 子时的隧穿电导相似, 但拉动 $\mathrm{CO}$ 分子的横向力却 为 $160 \mathrm{pN}$. 值得注意的是, 针尖与 $\mathrm{Co}$ 原子间的相互 作用力近乎球形对称, 而操纵 $\mathrm{CO}$ 分子的力严重偏
离球形对称. 作者在实验中测量了 $\mathrm{Co}$ 原子和 $\mathrm{CO}$ 分 子在表面不同方向的扩散势垒, 确定了原子在表面 不同吸附位的稳定性. 这些研究工作深入了人们对 分子在表面的扩散机制的认识, 并为在原子尺度上 的摩擦力研究开辟了新的道路. 最近, 清华大学的 陈䂀研究组 ${ }^{85}$ 定量测量了在 $\mathrm{Pb}(111)$ 表面横向移动 原酞菁 $\left(\mathrm{H}_{2} \mathrm{Pc}\right)$ 分子以及从 $\mathrm{H}_{2} \mathrm{Pc}$ 组装单层膜中拉出一 个分子的临界力的大小. 测量结果表明, 分子间相 互作用的横向临界力约为 $25 \mathrm{pN}$, 是分子与基底相 互作用的两倍.

平面有机分子接触电导的测量需要将分子操 纵成自由站立的构型, 如分子一端接触针尖, 另一 端接触基底. 有机分子一般通过多种作用吸附在金 属表面上, 如范德华相互作用、分子与金属的局域 化学键等. 从金属表面纵向提起有机分子会导致键 的断裂. 结合 STM 和 AFM, Temirov 与其合作者 ${ }^{86,87}$ 测量了从 $\operatorname{Ag}(111)$ 和 $\operatorname{Au}(111)$ 表面提起 PTCDA 分子 过程中的接触构型和电导的变化. 他们发现在 $\operatorname{Ag}(111)$ 表面操纵 PTCDA 分子时得到的谱线重复性 较差, 从 $\mathrm{Au}(111)$ 表面操纵分子时得到的谱线重复性 好. 这表明分子与 $\operatorname{Ag}(111)$ 表面之间断键有临界点, 是突然断裂的; 而分子与 $\mathrm{Au}(111)$ 表面之间键的断 裂是可逆的. Pawlak 等 ${ }^{88}$ 利用 NC-AFM 实现了机械 力引发的卟啉衍生物的可控旋转. 亚埃精度左右的 三维力谱表明, 只有分子的少数面积 $(\approx 100 \times 100$ $\mathrm{pm}^{2}$ ) 受到机械力作用分子才会转动. 分子受到局域 机械力作用时, 发生弹性形变, 从而导致分子的旋 转. 这一研究强调了复杂分子机械性质和局域反应 活性研究的重要性.

\section{4 原子尺度的电子性质分析方法}

原子尺度的接触为原子间成键过程、电子在分 子导线中的输运和摩擦力的微观机理等研究提供 了可能的途径. ${ }^{89,90}$ 原子尺度接触过程中电导/力与距 离的依赖关系得到了很多实验 ${ }^{91-94}$ 和理论 ${ }^{95,96}$ 研究的 关注. STM 研究发现原子尺度的电荷输运与针尖样品的接触结构密切相关. 接触过程中力与距离的 关系可以提供接触结构的信息. 结合 STM 和 AFM, 可以同时得到电荷输运和接触结构的信息. 2000 年, Schirmeisen 等 ${ }^{92}$ 首次同时测量了距离依赖的电 导和力的变化. 近几年原子尺度接触过程的距离依 赖的电导和力才得以精确测量. ${ }^{97-100}$ Sawada 等 ${ }^{97}$ 证 实针尖与 $\operatorname{Si}(111)-(7 \times 7)$ 表面处于近接触区域时观察 到的隧穿电流降低 ${ }^{101}$ 是针尖与 $\mathrm{Si}$ 增原子间形成化 
学键导致的. 2011 年, Ternes 等 ${ }^{98}$ 利用基于 qPlus 传 感器的 AFM 技术结合密度泛函理论(DFT)计算研 究了原子尺度的金属结成键过程中短程化学吸引 力以及隧穿电流随针尖-样品间距离以及不同材料 的变化. 他们发现短程力和电导随距离呈指数关系 衰减, 且衰减长度基本相同. 在隧穿至点接触的过 渡区域, 针尖与表面的短程力导致金属结结构的弛 豫并伴随表面电子结构的调节和隧穿势垒的妇塌. 这一工作表明原子尺度的金属结成键过程中的复 杂相互作用可以利用 NC-AFM 技术进行研究, 并且 和理论计算的结果相符. Hauptmann 等 ${ }^{100}$ 测量了 $\mathrm{Cu}-\mathrm{C}_{60}$ 和 $\mathrm{C}_{60}-\mathrm{C}_{60}$ 接触过程的电导和力, 他们发现 $\mathrm{C}_{60}-\mathrm{C}_{60}$ 接触的最大吸引力远小于 $\mathrm{Cu}-\mathrm{C}_{60}$ 的最大吸引 力. 之前 Schull 等 ${ }^{102,103}$ 报道的利用 STM 测量的 $\mathrm{Cu}-\mathrm{C}_{60}$ 和 $\mathrm{C}_{60}-\mathrm{C}_{60}$ 接触电导对应于张应力最大的接触.

单个原子/分子开关一般可以通过电子或者非 弹性共振隧穿、分子充电等方法实现. Sweetman 等 ${ }^{104}$ 利用 NC-AFM 的横向操纵技术展现了一种机械控 制双稳态体系开关的新方法. 这种开关机理可以非 破坏性地改变局域几何和电子结构. 他们在 $\operatorname{Si}(100)$ 表面利用针尖与样品之间的短程共价作用机械操 纵一个双稳态原子的键角变化, 实现了单个 buckled dimers 的翻转. Buckled dimers 的翻转会经历共 价键的断键和成键过程, 与目标原子的局域和非局 域环境密切相关, 这是未来原子尺度的加工过程需 要考虑的重要因素.

\section{5 基于原子尺度分辨率的开尔文探针力显微技术}

KPFM 是一种测量样品与导电探针间的接触势 差(CPD)的技术, 由 Nonnenmacher 等 ${ }^{105}$ 在 1991 年首 次提出. 基于平行金属板之间电容力的概念, $\mathrm{CPD}$ 的定义如下:

$$
V_{\mathrm{CPD}}=\frac{\Phi_{\text {sample }}-\Phi_{\text {tip }}}{|e|}
$$

其中, $\Phi_{\mathrm{tip}}$ 和 $\Phi_{\mathrm{sample}}$ 分别是针尖和样品的功函数, $e$ 是 电子的电量. 导电针尖接近样品表面时, 由于针尖 与样品功函数的不同, 二者之间会形成表观 $V_{\mathrm{CPD}}$, 产 生的静电相互作用可以通过在样品或者针尖上施 加偏压来抵消. KPFM 主要有两种工作模式: ${ }^{13}$ 电压 调制 KPFM 和 $\Delta f(V)$ 谱. 在电压调制 KPFM 中, 施加 在悬臂上的交流电压(频率为 $\omega_{0}$ )引发针尖 - 样品间 的静电力调制, 导致频率偏移 $\Delta f$ 在频率 $\omega_{0}$ 的振荡. 使 $\Delta f$ 振荡最小的直流偏压即为样品与导电探针之 间的 CPD. 一般而言, 频率偏移 $\Delta f$ 与偏压 $\left(V_{\text {bias }}\right)$ 的平
方成正比. $\Delta f(V)$ 曲线抛物线顶点对应的偏压即为样 品与导电探针间的 CPD, 所对应的 $\Delta f$ 为补偿静电力 梯度后的针尖-样品间相互作用力梯度. 在不同针 尖一样品间距 $z$ 处采集 $\Delta f(V)$ 谱, 可以得到 $\Delta f$ 随 $V_{\text {bias }}$ 和 $z$ 变化的三维谱图. ${ }^{106,107}$

CPD 的定义是基于平行金属板之间电容力的 概念, 本身并不包括原子尺度的径向静电力分布. 原子尺度分辨的 KPFM 测量的 $\mathrm{CPD}$ 被定义为局域 接触势差(LCPD). LCPD 来源于 Wandelt ${ }^{108,109}$ 提出的 局域功函数的概念. 局域功函数反映表面微观结构 如取向、电荷分布以及化学环境等因素对功函数的 影响. LCPD包括长程静电力和短程静电力的贡献. 离子晶体表面 LCPD 的原子分辨是由针尖尖端与表 面原子的短程静电作用力引起的. 对于绝缘体薄膜 上的单个原子电荷测量而言, 原子与针尖的相互作 用主要是范德华力、长程静电力和短程静电力. 其 中, 短程静电力对表面原子电荷的 AFM 成像起主要 作用, LCPD 的偏移则来自于长程静电力.

近年来, KPFM 的空间分辨率和能量分辨率得 到了显著提高. KPFM 已经得到了很多表面 LCPD的 原子分辨, ${ }^{110}$ 如 $\mathrm{Si}(111)-(7 \times 7) 、 \mathrm{Si}(111)-(5 \sqrt{3} \times 5 \sqrt{3})$ 、 $\mathrm{TiO}_{2}(110) 、 \mathrm{NaCl}$ 和 $\mathrm{KBr}$ 等. $\mathrm{KPFM}$ 可以在原子尺度 上以几个 $\mathrm{meV}$ 的能量分辨率对材料表面 LCPD 进 行成像. KPFM 具有单电子灵敏度, 可以检测量子点 的单电子充电等. 原子尺度的空间分辨率和单电子 灵敏度使得 KPFM 成为研究分子电子学、催化、光 伏电池和金属/有机界面的重要工具.

KPFM 可以区分金属原子的不同带电状态甚至 得到单个分子内的电荷分布. 2009 年, Gross 等 ${ }^{111}$ 使 用基于 qPlus 传感器的 KPFM, 测量了不同电荷状态 下金属原子与基底的接触势差, 即局域功函数, 区 分了金属原子的不同带电状态. 实验通过针尖施加 电压脉冲, 使得在 $\mathrm{NaCl}(2 \mathrm{ML}) / \mathrm{Cu}(111)$ 表面上沉积 的金属增原子(如 $\mathrm{Au}$ 和 $\mathrm{Ag}$ 原子)得到或失去一个电 子, 分别获得 $\mathrm{Au}^{-}$和 $\mathrm{Au}^{0}$ 以及 $\mathrm{Ag}^{+}$和 $\mathrm{Ag}^{0}$. 在金属增原 子上测量 $\Delta f(V)$ 谱, 并拟合抛物线得到曲线顶点所对 应的 LCPD, 发现由于带电状态的不同, 金属增原子 的 LCPD 偏移方向不同, 从而区分了金属增原子的 不同带电状态. 2012 年他们 ${ }^{12}$ 又利用三维 LCPD 成 像得到了单个互变异构的萗酞菁(naphthalocyanine) 分子 ${ }^{112}$ 内的电荷分布. 图 7(a) 是测量荎酞菁分子各 个位点 LCPD 的示意图. 图 7(b,c) 是萗酞菁分子互 变异构开关前后的 LCPD 亚分子分辨成像, 可以看 

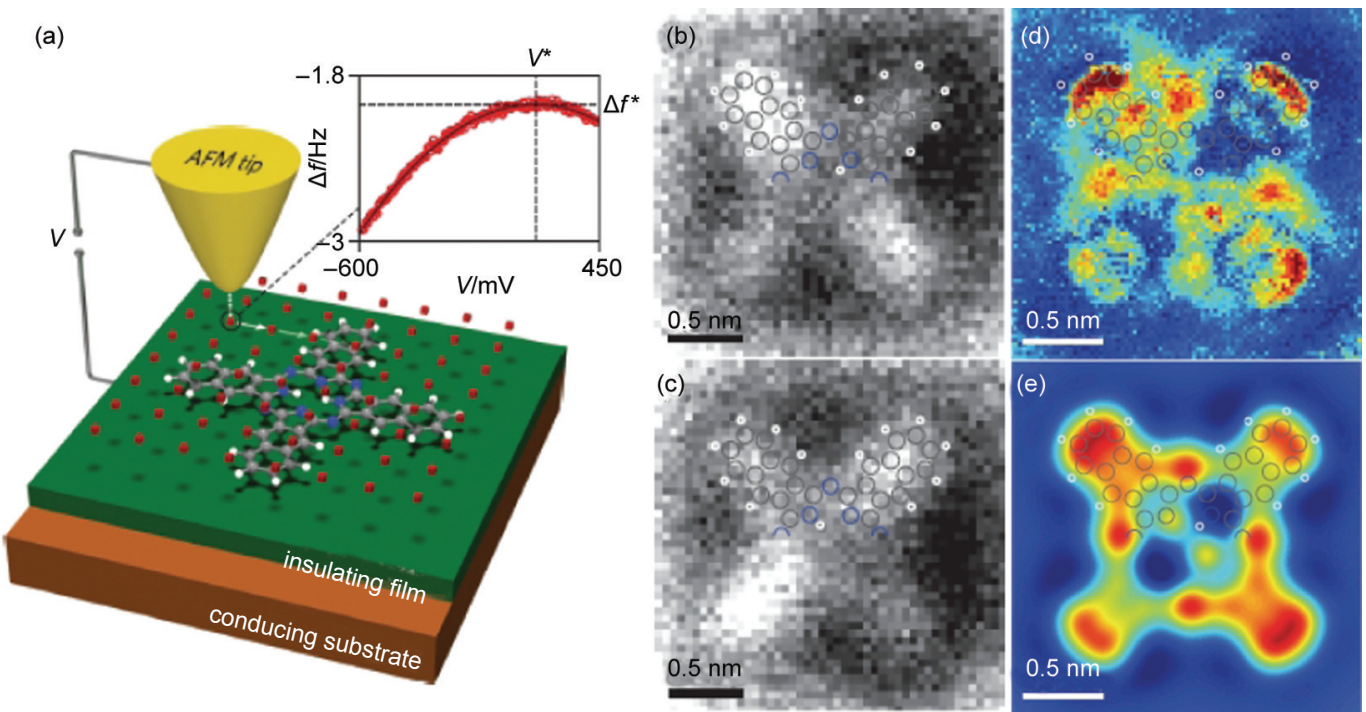

图 7 芸酞菁分子互变异构开关状态的 LCPD 成像 ${ }^{12}$

Fig.7 LCPD images of the tautomerization switching of naphthalocyanine ${ }^{12}$

(a) schematic illustration of the LCPD imaging and measurement principle. The frequency shift as a function of the sample bias voltage is recorded at each grid position (inset, red circles). The maximum of the fitted parabola (inset, solid black line) yields CPD for that position. (b, c) LCPD images of naphthalocyanine on $\mathrm{NaCl}(2 \mathrm{ML}) / \mathrm{Cu}(111)$ before and after switching the tautomerization state of the molecule, respectively. (d) LCPD images of a naphthalocyanine molecule recorded with a CO-terminated tip. (e) DFT-calculated $z$-component of the electric field above a free naphthalocyanine molecule at a distance $d=0.3 \mathrm{~nm}$ from the molecular plane. The DFT-calculated atomic positions are overlaid in the upper halves of (b)-(e). Carbon, hydrogen, and nitrogen atoms are in grey, white, and blue, respectively.

出分子中心含氮原子区域的 LCPD 高于含氢原子区 域的 LCPD. CO 针尖得到的 LCPD 图像分辨率更高 (图 7(d)). 结合理论模拟, 可以将 LCPD成像与分子 内的电荷分布联系在一起(图 7(d,e)). 这些工作可以 推广到多种材料表面, 为研究分子内及分子间电荷 重新分布的过程, 如分子开关、成键以及氧化还原 反应等, 提供了理论基础和研究的技术路径, 对单 分子化学反应、单电子器件和催化等研究有重要意 义.

Leoni 等 ${ }^{113}$ 结合 STM 和 KPFM 研究了一种新型 电机械分子开关的机理. 他们利用 STM 实现了吸附 在 $\mathrm{NaCl}(2 \mathrm{ML}) / \mathrm{Cu}(111)$ 表面上 bis-dibenzoylmethanato-copper $\left(\mathrm{Cu}(\mathrm{dbm})_{2}\right)$ 分子的两种稳态结构间的转 换, 并结合 KPFM确定了两种稳态结构的带电状 态, 指出稳态结构间的转换是分子充电引发的金属 配合物的结构重排. 之后他们 ${ }^{114}$ 将这一方法推广至 不同的金属有机化合物的研究.

Heyde 与其合作者 ${ }^{115}$ 利用 STM/AFM 双模式 SPM 系统研究了金属表面氧化物薄膜的缺陷的形 貌和电子结构. 他们 ${ }^{116}$ 利用 STS 技术测量了 $\operatorname{Ag}(100)$ 表面 $\mathrm{MgO}$ 薄膜的四种不同点缺陷的电子态结构, 并 利用 KPFM 测量了不同点缺陷的 LCPD. 不同点缺 陷 LCPD之间的差异表明, KPFM 可以区分不同的
点缺陷并对其进行分类. 结合 STM 和 AFM 对氧化 物薄膜的原子分辨成像以及 KPFM 对 LCPD 的测 量, 他们 ${ }^{117}$ 证实了理论预测的 $\mathrm{Ni} / \mathrm{Al}(110)$ 表面的氧化 铝薄膜反相畴界引起的线缺陷中似 $\mathrm{F}^{2+}$ 中心的存在. 与 STM 相比, KPFM 可以对广泛的材料(适用于绝 缘材料)进行表面结构和电特性的研究, 而且在绝缘 材料表面, 因长程静电相互作用力的影响很小, 有 可能进一步提高 KPFM 的电荷测量灵敏度.

金属/有机界面的性质是决定有机场效应晶体 管性能的重要因素. 金属电极上吸附有机分子自组 装薄膜能调节金属电极的功函数, 降低从有机半导 体薄膜中注入/提取载流子的能垒. Nikiforov 等 ${ }^{118}$ 利 用 KPFM研究了分子吸附取向对电极功函数的影 响. 他们发现卟啉配合物以两种吸附取向在 HOPG 上组装成岛状结构. 卟啉配合物单分子层以卟啉环 垂直于 HOPG 表面吸附时, 对 HOPG 功函数的调制 可以忽略, 而以卟啉环平行于 $\mathrm{HOPG}$ 表面吸附时, $\mathrm{HOPG}$ 与单分子层的 CPD 为 $-50 \mathrm{mV}$, 此研究说明, 除了吸附分子的化学结构, 其吸附构型也对有机/金 属界面的性质有重要的调控作用. Ichii 等 ${ }^{119}$ 得到了 $\mathrm{Au}(111)$ 表面铜酞菁 $\mathrm{CuPc}$ 单层膜分子分辨的 LCPD 成像. LCPD成像反差来源于分子/电极间的表面偶 极. Glatzel ${ }^{110}$ 研究了吐昔烯(truxene)分子在 $\mathrm{KBr}$ 表面 
的自组装. 他们得到了 truxene 分子亚分子分辨的 LCPD 成像. Truxene 分子的 LCPD 比基底低 200 $\mathrm{mV}$, 与分子偶极取向一致. 但是单分子分辨的 LCPD 强烈依赖于针尖的形状和成分, 其定量分析 仍具有较大的挑战. 在单分子分辨的 LCPD数据的 定量分析中, 需考虑针尖的形状和成分以及可能的 空间平均化效应.

\section{4 总结与展望}

$\mathrm{NC}-\mathrm{AFM}$ 是一个高速发展的新兴领域, 尤其是 近几年来 qPlus 传感器的应用使得 NC-AFM 在成像 分辨率、原子/分子操纵等方面可与 STM 婫美. qPlus 传感器使得 NC-AFM 的振幅进入了亚埃量级, 力的 检测对短程化学力极度敏感, 从而能够得到分子内 的原子分辨图像. NC-AFM 自身功能的拓展及其与 不同探针技术的联用拓宽了研究领域. NC-AFM 与 STM 可以研究不同的物理、化学特性, 二者的联用 已经成为全面而深入地研究原子尺度接触问题不 可或缺的工具. 其衍生技术 KPFM 已经成为一种具 有高空间分辨和能量分辨的表征手段, 可以同时获 得表面形貌和表面电势的信息, 使其成为研究纳米 结构电特性的理想工具. 原子/分子操纵、NC-AFM 与 STM 的谱学成像以及 KPFM 的单电子检测功能 相结合, 可以在表面构造功能纳米结构, 并研究分 子内电荷分布、电荷传输路径和化学反应活性等性 质, 为材料、物理、化学和生命科学有关的研究提供 了崭新的思路.

尽管 NC-AFM 已经取得了飞速的进步, 但其也 有技术上的局限性. 从扫描成像和记录信号的角度 考虑, NC-AFM 仍然是一种慢成像技术, 较低的时 间分辨率限制了其在跟踪表面分子扩散、化学反应 等快过程方面的研究. 开发高频力传感器, 如长度 伸缩模石英谐振器 ${ }^{22,23}$ 有利于这一问题的解决. 恒高 模式可以获得比恒频率偏移模式更快的成像速度, 如共振频率为 $300 \mathrm{kHz}$ 悬臂, 其恒高模式成像速度 高达 $60 \mathrm{~Hz}$, 这为 NC-AFM 开启了快速成像的大 门. ${ }^{120}$ 而 NC-AFM 与具有皮秒精度和自旋灵敏的动 态 $\mathrm{STM}^{121}$ 的联用, 将为振动激发态、分子构型转变 等随时间的演化提供新的视角. NC-AFM 高分辨成 像的关键是针尖的修饰, 目前主要依靠惰性分子, 这局限了 NC-AFM 的对化学反应活性和机理的研 究. 利用真空蒸镀、分子操纵以及溶液修饰等方法 开发不同化学性质的探针, 可以在原子尺度上深入
理解化学反应机理以及环境的影响.

\section{References}

(1) Binnig, G.; Rohrer, H.; Gerber, C.; Weibel, E. Phys. Rev. Lett. 1982, 49, 57. doi: 10.1103/PhysRevLett.49.57

(2) Teague, E. C. Bull. Am. Phys. Soc. 1978, 23, 290.

(3) Coombs, J. H.; Pethica, J. B. IBM J. Res. Dev. 1986, 30, 455. doi: $10.1147 /$ rd.305.0455

(4) Binnig, G.; Quate, C. F.; Gerber, C. Phys. Rev. Lett. 1986, 56, 930. doi: 10.1103/PhysRevLett.56.930

(5) García, R.; Pérez, R. Surf. Sci. Rep. 2002, 47, 197. doi: 10.1016/ S0167-5729(02)00077-8

(6) Martin, Y.; Williams, C. C.; Wickramasinghe, H. K. J. Appl. Phys. 1987, 61, 4723. doi: 10.1063/1.338807

(7) Zhong, Q.; Inniss, D.; Kjoller, K.; Elings, V. B. Surf. Sci. Lett. 1993, 290, L688.

(8) Albrecht, T. R.; Grütter, P.; Horne, D.; Rugar, D. J. Appl. Phys. 1991, 69, 668. doi: 10.1063/1.347347

(9) Gross, L. Nat. Chem. 2011, 3, 273. doi: 10.1038/nchem.1008

(10) Gross, L.; Mohn, F.; Moll, N.; Schuler, B.; Criado, A.; Guitián, E.; Peña, D.; Gourdon, A.; Meyer, G. Science 2012, 337, 1326. doi: $10.1126 /$ science. 1225621

(11) Baykara, M. Z.; Schwendemann, T. C.; Altman, E. I.; Schwarz, U. D. Adv. Mater. 2010, 22, 2838. doi: 10.1002/adma.200903909

(12) Mohn, F.; Gross, L.; Moll, N.; Meyer, G. Nat. Nanotechnol. 2012, 7, 227. doi: 10.1038/nnano.2012.20

(13) Melitz, W.; Shen, J.; Kummel, A. C.; Lee, S. Surf. Sci. Rep. 2011, 66, 1. doi: 10.1016/j.surfrep.2010.10.001

(14) Barth, C.; Foster, A. S.; Henry, C. R.; Shluger, A. L. Adv. Mater. 2011, 23, 477. doi: 10.1002/adma.v23.4

(15) Giessibl, F. J. Rev. Mod. Phys. 2003, 75, 949. doi: 10.1103/ RevModPhys.75.949

(16) Pérez, R.; Štich, I.; Payne, M. C.; Terakura, K. Phys. Rev. B 1998, 58, 10835. doi: 10.1103/PhysRevB.58.10835

(17) Livshits, A. I.; Shluger, A. L.; Rohl, A. L.; Foster, A. S. Phys. Rev. B 1999, 59, 2436. doi: 10.1103/PhysRevB.59.2436

(18) Moll, N.; Gross, L.; Mohn, F.; Curioni, A.; Meyer, G. N. J. Phys. 2010, 12, 125020. doi: 10.1088/1367-2630/12/12/125020

(19) Moll, N.; Gross, L.; Mohn, F.; Curioni, A.; Meyer, G. N. J. Phys. 2012, 14, 083023. doi: 10.1088/1367-2630/14/8/083023

(20) Custance, Ó.; Oyabu, N.; Sugimoto, Y. Force Spectroscopy on Semiconductor Surfaces. In Noncontact Atomic Force Microscopy; Morita, S., Giessibl, F. J., Wiesendanger, R. Eds.; Springer: Berlin, 2009; Vol. 2, pp 31-68.

(21) Giessibl, F. J. Materials Today 2005, 8, 32.

(22) Torbrugge, S.; Schaff, O.; Rychen, J. J. Vac. Sci. Technol. B 2010, 28, C4E12.

(23) An, T.; Nishio, T.; Eguchi, T.; Ono, M.; Nomura, A.; Akiyama, K.; Hasegawa, Y. Rev. Sci. Instrum. 2008, 79, 033703. doi: $10.1063 / 1.2830937$ 
(24) Giessibl, F. J. Appl. Phys. Lett. 1998, 73, 3956. doi: 10.1063/ 1.122948

(25) Heyde, M.; Kulawik, M.; Rust, H. P.; Freund, H. J. Rev. Sci. Instrum. 2004, 75, 2446. doi: 10.1063/1.1765753

(26) Giessibl, F. J.; Pielmeier, F.; Eguchi, T.; An, T.; Hasegawa, Y. Phys. Rev. B 2011, 84, 125409.

(27) Giessibl, F. J. Principles and Applications of the qPlus Sensor. In Noncontact Atomic Force Microscopy; Morita, S., Giessibl, F. J., Wiesendanger, R. Eds.; Springer: Berlin, 2009; Vol. 2, pp 121-142.

(28) Sader, J. E.; Jarvis, S. P. Appl. Phys. Lett. 2004, 84, 1801. doi: 10.1063/1.1667267

(29) Hamaker, H. C. Physica 1937, 4, 1058. doi: 10.1016/S0031-8914 (37)80203-7

(30) Israelachvili, J. Intermolecular and Surface Forces, 3rd ed.; Academic Press: San Diego, 2011.

(31) Argento, C.; French, R. H. J. Appl. Phys. 1996, 80, 6081. doi: $10.1063 / 1.363680$

(32) Ruschmeier, K.; Schirmeisen, A.; Hoffmann, R. Phys. Rev. Lett. 2008, 101, 156102. doi: 10.1103/PhysRevLett.101.156102

(33) Such, B.; Glatzel, T.; Kawai, S.; Koch, S.; Meyer, E. J. Vac. Sci. Technol. B 2010, 28, C4B1.

(34) Kawai, S.; Glatzel, T.; Koch, S.; Baratoff, A.; Meyer, E. Phys. Rev. B 2011, 83, 035421. doi: 10.1103/PhysRevB.83.035421

(35) Sun, Z. X.; Boneschanscher, M. P.; Swart, I.; Vanmaekelbergh, D.; Liljeroth, P. Phys. Rev. Lett. 2011, 106, 046104. doi:10.1103/ PhysRevLett.106.046104

(36) Fremy, S.; Kawai, S.; Pawlak, R.; Glatzel, T.; Baratoff, A.; Meyer, E. Nanotechnology 2012, 23, 055401. doi: 10.1088/ 0957-4484/23/5/055401

(37) Albers, B. J.; Schwendemann, T. C.; Baykara, M. Z.; Pilet, N.; Liebmann, M.; Altman, E. I.; Schwarz, U. D. Nanotechnology 2009, 20, 264002. doi: 10.1088/0957-4484/20/26/264002

(38) Albers, B. J.; Schwendemann, T. C.; Baykara, M. Z.; Pilet, N.; Liebmann, M.; Altman, E. I.; Schwarz, U. D. Nat. Nanotechnol. 2009, 4, 307. doi: 10.1038/nnano.2009.57

(39) Weiss, C.; Wagner, C.; Temirov, R.; Tautz, F. S. J. Am. Chem. Soc. 2010, 132, 11864. doi: 10.1021/ja104332t

(40) Temirov, R.; Soubatch, S.; Neucheva, O.; Lassise, A. C.; Tautz, F. S. N. J. Phys. 2008, 10, 053012. doi: 10.1088/1367-2630/10/ $5 / 053012$

(41) Weiss, C.; Wagner, C.; Kleimann, C.; Rohlfing, M.; Tautz, F. S.; Temirov, R. Phys. Rev. Lett. 2010, 105, 086103. doi: 10.1103/ PhysRevLett.105.086103

(42) Kichin, G.; Weiss, C.; Wagner, C.; Tautz, F. S.; Temirov, R. J. Am. Chem. Soc. 2011, 133, 16847. doi: 10.1021/ja204624g

(43) Giessibl, F. J. Science 1995, 267, 68. doi: 10.1126/science. 267.5194.68

(44) Noncontact Atomic Force Microscopy; Morita, S., Wiesendanger, R., Meyer, E. Eds.; Springer: Berlin, 2002; Vol. 1.

(45) Morita, S. Introduction. In Noncontact Atomic Force
Microscopy; Morita, S., Giessibl, F. J., Wiesendanger, R. Eds.; Springer: Berlin, 2009; Vol. 2; pp 1-13.

(46) Giessibl, F. J.; Hembacher, S.; Bielefeldt, H.; Mannhart, J. Science 2000, 289, 422. doi: 10.1126/science.289.5478.422

(47) Hembacher, S.; Giessibl, F. J.; Mannhart, J. Science 2004, 305, 380. doi: 10.1126/science. 1099730

(48) Gross, L.; Mohn, F.; Moll, N.; Liljeroth, P.; Meyer, G. Science 2009, 325, 1110. doi: 10.1126/science. 1176210

(49) Pawlak, R.; Kawai, S.; Fremy, S.; Glatzel, T.; Meyer, E. ACS Nano 2011, 5, 6349. doi: 10.1021/nn201462g

(50) Pawlak, R.; Kawai, S.; Fremy, S.; Glatzel, T.; Meyer, E. J. Phys. Condes. Matter 2012, 24, 084005. doi: 10.1088/0953-8984/24/8/ 084005

(51) Boneschanscher, M. P.; van der Lit, J.; Sun, Z.; Swart, I.; Liljeroth, P.; Vanmaekelbergh, D. ACS Nano 2012, 6, 10216. doi: $10.1021 / \mathrm{nn} 3040155$

(52) Pérez, R. Science 2012, 337, 1305. doi: 10.1126/science. 1227726

(53) Gross, L.; Mohn, F.; Moll, N.; Meyer, G.; Ebel, R.; AbdelMageed, W. M.; Jaspars, M. Nat. Chem. 2010, 2, 821. doi: $10.1038 /$ nchem.765

(54) Welker, J.; Giessibl, F. J. Science 2012, 336, 444. doi: 10.1126/ science. 1219850

(55) Mohn, F.; Repp, J.; Gross, L.; Meyer, G.; Dyer, M. S.; Persson, M. Phys. Rev. Lett. 2010, 105, 266102. doi: 10.1103/Phys RevLett.105.266102

(56) Pavliček, N.; Fleury, B.; Neu, M.; Niedenführ, J.; HerranzLancho, C.; Ruben, M.; Repp, J. Phys. Rev. Lett. 2012, 108 , 086101. doi: 10.1103/PhysRevLett.108.086101

(57) Stipe, B. C.; Rezaei, M. A.; Ho, W. Science 1998, 280, 1732. doi: $10.1126 /$ science. 280.5370 .1732

(58) Setvín, M.; Mutombo, P.; Ondrácek, M.; Majzik, Z.; Švec, M.; Cháb, V.; Oštádal, I.; Sobotík, P.; Jelínek, P. ACS Nano 2012, 6, 6969. doi: 10.1021/nn301996k

(59) Sugimoto, Y.; Pou, P.; Abe, M.; Jelinek, P.; Perez, R.; Morita, S.; Custance, Ó. Nature 2007, 446, 64. doi: 10.1038/nature05530

(60) Hoffmann, R.; Kantorovich, L. N.; Baratoff, A.; Hug, H. J.; Güntherodt, H. J. Phys. Rev. Lett. 2004, 92, 146103. doi: 10.1103/PhysRevLett.92.146103

(61) Lantz, M. A.; Hoffmann, R.; Foster, A. S.; Baratoff, A.; Hug, H. J.; Hidber, H. R.; Güntherodt, H. J. Phys. Rev. B 2006, 74, 245426. doi: 10.1103/PhysRevB.74.245426

(62) Foster, A. S.; Barth, C.; Henry, C. R. Phys. Rev. Lett. 2009, 102, 256103. doi: 10.1103/PhysRevLett.102.256103

(63) Hoffmann, R.; Weiner, D.; Schirmeisen, A.; Foster, A. S. Phys. Rev. B 2009, 80, 115426. doi: 10.1103/PhysRevB.80.115426

(64) Lantz, M. A.; Hug, H. J.; Hoffmann, R.; van Schendel, P. J. A.; Kappenberger, P.; Martin, S.; Baratoff, A.; Güntherodt, H. J. Science 2001, 291, 2580. doi: 10.1126/science.1057824

(65) Guo, C. S.; Van Hove, M. A.; Zhang, R. Q.; Minot, C. Langmuir 2010, 26, 16271. doi: 10.1021/la101317s 
(66) Eigler, D. M.; Schweizer, E. K. Nature 1990, 344, 524. doi: $10.1038 / 344524 \mathrm{a} 0$

(67) Bartels, L.; Meyer, G.; Rieder, K. H. Phys. Rev. Lett. 1997, 79, 697. doi: 10.1103/PhysRevLett.79.697

(68) Bartels, L.; Meyer, G.; Rieder, K. H. Appl. Phys. Lett. 1997, 71, 213. doi: $10.1063 / 1.119503$

(69) Eigler, D. M.; Lutz, C. P.; Rudge, W. E. Nature 1991, 352, 600. doi: $10.1038 / 352600 \mathrm{a} 0$

(70) Crommie, M. F.; Lutz, C. P.; Eigler, D. M. Science 1993, 262, 218. doi: $10.1126 /$ science. 262.5131 .218

(71) Yamachika, R.; Grobis, M.; Wachowiak, A.; Crommie, M. F. Science 2004, 304, 281. doi: 10.1126/science.1095069

(72) Custance, Ó.; Pérez, R.; Morita, S. Nat. Nanotechnol. 2009, 4, 803. doi: $10.1038 /$ nnano.2009.347

(73) Tseng, A. A. Nano Today 2011, 6, 493. doi: 10.1016/j.nantod. 2011.08 .003

(74) Oyabu, N.; Custance, Ó.; Yi, I.; Sugawara, Y.; Morita, S. Phys. Rev. Lett. 2003, 90, 176102. doi: 10.1103/PhysRevLett. 90.176102

(75) Sugimoto, Y.; Jelinek, P.; Pou, P.; Abe, M.; Morita, S.; Custance, Ó.; Pérez, R. Phys. Rev. Lett. 2007, 98, 106104. doi: 10.1103/ PhysRevLett.98.106104

(76) Oyabu, N.; Sugimoto, Y.; Abe, M.; Custance, Ó.; Morita, S. Nanotechnology 2005, 16, S112.

(77) Sugimoto, Y.; Abe, M.; Hirayama, S.; Oyabu, N.; Custance, Ó.; Morita, S. Nat. Mater. 2005, 4, 156. doi: 10.1038/nmat1297

(78) Sugimoto, Y.; Custance, Ó.; Abe, M.; Morita, S. e-J. Surf. Sci. Nanotech. 2006, 4, 376. doi: 10.1380/ejssnt.2006.376

(79) Sugimoto, Y.; Pou, P.; Custance, Ó.; Jelinek, P.; Abe, M.; Pérez, R.; Morita, S. Science 2008, 322, 413. doi: 10.1126/science. 1160601

(80) Swart, I.; Sonnleitner, T.; Niedenführ, J.; Repp, J. Nano Lett. 2012, 12, 1070. doi: 10.1021/n1204322r

(81) Hirth, S.; Ostendorf, F.; Reichling, M. Nanotechnology 2006, $17, \mathrm{~S} 148$

(82) Nishi, R.; Miyagawa, D.; Seino, Y.; Yi, I.; Morita, S. Nanotechnology 2006, 17, S142.

(83) Yi, I.; Nishi, R.; Abe, M.; Sugimoto, Y.; Morita, S. Jpn. J. Appl. Phys. Lett. 2011, 50, 015201. doi: 10.1143/JJAP.50.015201

(84) Ternes, M.; Lutz, C. P.; Hirjibehedin, C. F.; Giessibl, F. J.; Heinrich, A. J. Science 2008, 319, 1066. doi: 10.1126/science. 1150288

(85) Mao, H. Q.; Li, N.; Chen, X.; Xue, Q. K. J. Phys. Condes. Matter 2012, 24, 084004. doi: 10.1088/0953-8984/24/8/084004

(86) Fournier, N.; Wagner, C.; Weiss, C.; Temirov, R.; Tautz, F. S. Phys. Rev. B 2011, 84, 035435. doi: 10.1103/PhysRevB. 84.035435

(87) Wagner, C.; Fournier, N.; Tautz, F. S.; Temirov, R. Phys. Rev. Lett. 2012, 109, 076102. doi: 10.1103/PhysRevLett.109.076102

(88) Pawlak, R.; Fremy, S.; Kawai, S.; Glatzel, T.; Fang, H.; Fendt, L. A.; Diederich, F.; Meyer, E. ACS Nano 2012, 6, 6318. doi: $10.1021 / \mathrm{nn} 301774$ d

(89) Zhao, J. W.; Liu, H. M.; Ni, W. B.; Guo, Y.; Yin, X. Acta Phys. -Chim. Sin. 2009, 25, 1472. [赵建伟, 刘洪梅, 倪文涁, 郭 彦, 尹 星. 物理化学学报, 2009, 25, 1472.] doi: 10.3866/ PKU.WHXB20090744

(90) Ai, Y.; Zhang, H. L. Acta Phys. -Chim. Sin. 2012, 28, 2237. [艾 勇, 张浩力. 物理化学学报, 2012, 28, 2237.] doi: 10.3866/PKU.WHXB201209102

(91) Rubio-Bollinger, G.; Joyez, P.; Agraït, N. Phys. Rev. Lett. 2004, 93, 116803. doi: 10.1103/PhysRevLett.93.116803

(92) Schirmeisen, A.; Cross, G.; Stalder, A.; Grütter, P.; Dürig, U. N. J. Phys. 2000, 2, 29. doi: 10.1088/1367-2630/2/1/329

(93) Sun, Y.; Mortensen, H.; Schär, S.; Lucier, A. S.; Miyahara, Y.; Grütter, P.; Hofer, W. Phys. Rev. B 2005, 71, 193407. doi: 10.1103/PhysRevB.71.193407

(94) Hembacher, S.; Giessibl, F. J.; Mannhart, J.; Quate, C. F. Phys. Rev. Lett. 2005, 94, 056101. doi: 10.1103/PhysRevLett. 94.056101

(95) Chen, C. J. Nanotechnology 2005, 16, S27.

(96) Hofer, W. A.; Fisher, A. J. Phys. Rev. Lett. 2003, 91, 036803. doi: 10.1103/PhysRevLett.91.036803

(97) Sawada, D.; Sugimoto, Y.; Morita, K. I.; Abe, M.; Morita, S. Appl. Phys. Lett. 2009, 94, 173117. doi: 10.1063/1.3127503

(98) Ternes, M.; González, C.; Lutz, C. P.; Hapala, P.; Giessibl, F. J.; Jelínek, P.; Heinrich, A. J. Phys. Rev. Lett. 2011, 106, 016802. doi: 10.1103/PhysRevLett.106.016802

(99) Majzik, Z.; Setvín, M.; Bettac, A.; Feltz, A.; Cháb, V.; Jelínek, P. Beilstein J. Nanotechnol. 2012, 3, 249. doi: 10.3762/ bjnano. 3.28

(100) Hauptmann, N.; Mohn, F.; Gross, L.; Meyer, G.; Frederiksen, T.; Berndt, R. N. J. Phys. 2012, 14, 073032. doi: 10.1088/ $1367-2630 / 14 / 7 / 073032$

(101) Jelínek, P.; Švec, M.; Pou, P.; Pérez, R.; Cháb, V. Phys. Rev. Lett. 2008, 101, 176101. doi: 10.1103/PhysRevLett.101.176101

(102) Schull, G.; Frederiksen, T.; Brandbyge, M.; Berndt, R. Phys. Rev. Lett. 2009, 103, 206803. doi: 10.1103/PhysRevLett. 103.206803

(103) Schull, G.; Dappe, Y. J.; González, C. S.; Bulou, H.; Berndt, R. Nano Lett. 2011, 11, 3142. doi: 10.1021/n1201185y

(104) Sweetman, A.; Jarvis, S.; Danza, R.; Bamidele, J.; Gangopadhyay, S.; Shaw, G. A.; Kantorovich, L.; Moriarty, P. Phys. Rev. Lett. 2011, 106, 136101. doi: 10.1103/PhysRevLett. 106.136101

(105) Nonnenmacher, M.; O'Boyle, M. P.; Wickramasinghe, H. K. Appl. Phys. Lett. 1991, 58, 2921. doi: 10.1063/1.105227

(106) Sadewasser, S.; Jelinek, P.; Fang, C. K.; Custance, Ó.; Yamada, Y.; Sugimoto, Y.; Abe, M.; Morita, S. Phys. Rev. Lett. 2009, 103, 266103. doi: 10.1103/PhysRevLett.103.266103

(107) König, T.; Heinke, L.; Simon, G. H.; Heyde, M. Phys. Rev. B 2011, 83, 195435. doi: 10.1103/PhysRevB.83.195435

(108) Küppers, J.; Wandelt, K.; Ertl, G. Phys. Rev. Lett. 1979, 43, 
928. doi: 10.1103/PhysRevLett.43.928

(109) Wandelt, K. Appl. Surf. Sci. 1997, 111, 1. doi: 10.1016/S01694332(96)00692-7

(110) Glatzel, T. Measuring Atomic-Scale Variations of the Electrostatic Force. In Kelvin Probe Force Microscopy; Sadewasser, S., Glatzel, T. Eds.; Springer: Berlin, 2012; pp 289-327.

(111) Gross, L.; Mohn, F.; Liljeroth, P.; Repp, J.; Giessibl, F. J.; Meyer, G. Science 2009, 324, 1428. doi: 10.1126/science. 1172273

(112) Liljeroth, P.; Repp, J.; Meyer, G. Science 2007, 317, 1203. doi: $10.1126 /$ science. 1144366

(113) Leoni, T.; Guillermet, O.; Walch, H.; Langlais, V.; Scheuermann, A.; Bonvoisin, J.; Gauthier, S. Phys. Rev. Lett. 2011, 106, 216103. doi: 10.1103/PhysRevLett.106.216103

(114) Walch, H.; Leoni, T.; Guillermet, O.; Langlais, V.; Scheuermann, A.; Bonvoisin, J.; Gauthier, S. Phys. Rev. B 2012, 86, 075423. doi: 10.1103/PhysRevB.86.075423

(115) König, T.; Simon, G. H.; Heinke, L.; Lichtenstein, L.; Heyde,
M. Beilstein J. Nanotechnol. 2011, 2, 1. doi: 10.3762/bjnano. 2.1

(116) König, T.; Simon, G. H.; Rust, H. P.; Pacchioni, G.; Heyde, M.; Freund, H. J. J. Am. Chem. Soc. 2009, 131, 17544. doi: $10.1021 /$ ja 908049 n

(117) Heinke, L.; Lichtenstein, L.; Simon, G. H.; König, T.; Heyde, M.; Freund, H. J. ChemPhysChem 2010, 11, 2085. doi: 10.1002/cphc.v11:10

(118) Nikiforov, M. P.; Zerweck, U.; Milde, P.; Loppacher, C.; Park, T. H.; Uyeda, H. T.; Therien, M. J.; Eng, L.; Bonnell, D. Nano Lett. 2008, 8, 110. doi: 10.1021/n1072175d

(119) Ichii, T.; Fukuma, T.; Yoda, T.; Kobayashi, K.; Matsushige, K.; Yamada, H. J. Appl. Phys. 2010, 107, 024315. doi: 10.1063/ 1.3284094

(120) Barth, C.; Pakarinen, O. H.; Foster, A. S.; Henry, C. R. Nanotechnology 2006, 17, S128.

(121) Loth, S.; Etzkorn, M.; Lutz, C. P.; Eigler, D. M.; Heinrich, A. J. Science 2010, 329, 1628. doi: 10.1126/science. 1191688 